\title{
CLIMATE VARIABILITY AND MALARIA INCIDENCE IN PERI-URBAN, URBAN AND RURAL COMMUNITIES AROUND KUMASI, GHANA: A CASE STUDY AT THREE HEALTH FACILITIES; EMENA, ATONSU AND AKROPONG
}

\author{
TAY S.C.K. ${ }^{*}$, DANUOR S.K. ${ }^{1}$, MENSAH D.C. ${ }^{1}$, ACHEAMPONG G. ${ }^{1}$, ABRUQUAH H.H. ${ }^{2}$, MORSE A. ${ }^{3}$, \\ CAMINADE C. ${ }^{3}$, BADU K. ${ }^{1,4}$, TOMPKINS A. ${ }^{5}$ AND HASSAN H.A. ${ }^{6}$
}

${ }^{1}$ Kwame Nkrumah University of Science and Technology (KNUST), Kumasi, Ghana.

2University Hospital, KNUST, Kumasi, Ghana.

3University of Liverpool, Liverpool, L69 3BX, United Kingdom.

${ }^{4}$ Climate and Human Health Research Unit, Center for Global Health Research, Kenya Medical Research Institute, Kisumu, Kenya.

${ }^{5}$ Abdus Salam International Centre for Theoretical Physics, Trieste, Italy.

6University Library, KNUST, Ghana.

*Corresponding Author: Email- scktaysammy9@yahoo.com

Received: June 28, 2012; Accepted: July 06, 2012

\begin{abstract}
-
Background: Changes in temperature, rainfall and relative humidity due to climate change are expected to directly influence malaria transmission dynamics, by modifying the behavior and geographical distribution of malaria vectors while shortening the sporogonic cycle of the parasite in the vector. These changes will have varying effects on malaria incidence in different eco-epidemiological settings. The aim was to examine the impact of climate variability on malaria incidence in three micro-epidemiological ecosystems in the forest zone of Ghana, Ashanti Region.

Methods: Data on climatic variables (rainfall, temperature and humidity) from January 2008 to- October 2009 were obtained from the two weather stations. Hospital based confirmed malaria incidence data were obtained for the same period from Akropong (rural) hospital whereas malaria incidence data were obtained from January 2008-June 2009 and January 2008-August 2009 for Emena (peri-urban) and Atonsu (urban) hospitals respectively. Microscopy was used to identify the predominant Plasmodium species. Spearman's correlation analysis and cross correlation analysis that allowed a time-lag of 0-3 month were performed to examine the role of climatic variables on malaria incidence.

Results: There was a direct correlation between monthly malaria incidence and monthly climatic variables at different time-lags and location. At every study site, different climatic variables were seen to play different roles on the occurrence of malaria. At Akropong and Emena, the study indicates that rainfall and minimum temperature are the major drivers of malaria incidence though this relationship was significant at different time-lags in different eco-epidemiological settings. At Atonsu, the study indicates that rainfall and maximum temperature are the major contributors to malaria incidence.

Conclusion: Climatic variables have direct and indirect influence on malaria incidence and these variables can predict malaria incidence and be suited for forecasting the malaria occurrence in areas with similar ecological features in the Ashanti Region of Ghana.
\end{abstract}

Key words- Climate variability, malaria, Plasmodium species.

Citation: Tay S.C.K., et al. (2012) Climate Variability and Malaria Incidence in Peri-urban, Urban and Rural Communities Around Kumasi, Ghana: A Case Study at Three Health Facilities; Emena, Atonsu and Akropong. International Journal of Parasitology Research, ISSN: 09753702 \& E-ISSN: 0975-9182, Volume 4, Issue 2, pp.-83-89.

Copyright: Copyright@2012 Tay S.C.K., et al. This is an open-access article distributed under the terms of the Creative Commons Attribution License, which permits unrestricted use, distribution and reproduction in any medium, provided the original author and source are credited.

\section{Background}

Malaria remains one of the most important causes of human morbidity and mortality with significant medical and economic impact on the world [1]. WHO found limited evidence of a decrease in malaria burden in Ghana in recent years, despite declining malaria burden in other parts of Africa [3]. In Ghana, particularly in the forest zone, there is perennial transmission of malaria with highest intensity during rainy season [4], accounting for about $32-42 \%$ of all outpatient admissions and for major inpatient causes of death [5]. 
It is known to be among the top four leading causes of death in children under five and pregnant women in developing countries [2].

Malaria has shown to be a complex disease and its transmission and prevalence influenced by many factors, amongst which variability in climatic factors play a major role. Increases in temperature, rainfall and relative humidity due to climate change are expected to influence malaria directly by enhancing the survival and geographical distribution of malaria vectors and by shortening the length of the life cycle of the parasite within the vector [6]. This is because temperature affects the development rates and survivorship of malaria parasites and mosquito vectors, whilst rainfall influences the availability of mosquito larval habitats and thus mosquito demography and behavior [7]

Several studies have linked incidence of malaria time series to weather variables such as rainfall, temperature and humidity. Whereas some found strong correlation between malaria and climatic variables [8-10], others did not find any significant relationship $[11,12]$.

One study in particular from the Ashanti region of Ghana reported that, high-resolution precipitation data can directly predict malaria incidence in a highly endemic area within a mean interval of two month lag [13].

It has been reported that the synergistic effect of temperature and rainfall on malaria transmission is much more pronounced than individual effects [14]. Thus, simultaneous analysis of meteorological and parasitological data at different micro-epidemiological ecosystems at the local level is critically needed to demonstrate the effects of climate on malaria cases.

However, there is limited information on the association between climate variability and empirical malaria incidence in Ghana. Thus there is the need to rigorously examine this association from different transmission and eco-epidemiological zones. The knowledge will be critical to the development of malaria early warning systems in the forest zone of Ghana and other similar regional epidemiological ecosystems. The purpose of the study was therefore to provide a baseline data to enhance a future malaria control program, develop forecasting and early warning system by linking climate variables and malaria occurrence in Ghana

\section{Materials and Methods}

\section{Study Design}

This is a longitudinal study that employed both retrospective and prospective data to determine the impact of climate variables (rainfall, relative humidity and temperature) on malaria incidence across three micro-epidemiological ecosystems; in urban (Atonsu), peri-urban (Emena) and rural (Akropong) districts of the Ashanti region of Ghana. The study population included all cases of suspected malaria referred to the laboratory for screening.

\section{Study Area}

This study was conducted in the Kumasi Metropolis (Fig. 1); including Atonsu, a suburb and Emena, a peri urban area. The third site, Akropong is a rural district in the Ashanti Region where Kumasi is the capital. Kumasi is the second largest city in Ghana, with a pop- ulation of about 2.5 million [15] and is located between latitude $30^{\prime \prime}$ and $7^{\circ} 00^{\prime \prime} \mathrm{N}$ and longitude $1^{\circ} 30^{\prime \prime}$ and $2^{\circ} 00^{\prime \prime} \mathrm{W}$ and covers an area of $24,389 \mathrm{sq}$. $\mathrm{km}$. The region is located in the central forest zone of Ghana with a semi-humid wet tropical climate. Annual rainfall is about $1400 \mathrm{~mm}$ with two distinct rainy seasons. The mean annual temperature is $25.7^{\circ} \mathrm{C}$ with a humidity ranging from 53 to $93 \%$. Malaria is hyper-endemic in this region; often recording the highest number of cases in the country.

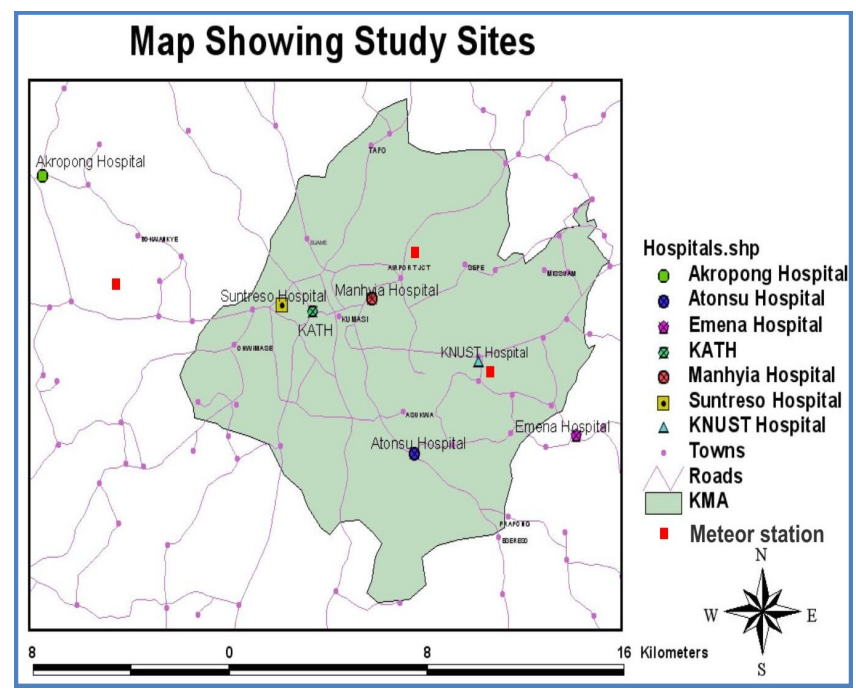

Fig. 1- Map of Study Site

\section{Akropong}

Akropong is a rural community located between latitude $6^{\circ} 74^{\prime} 17^{\prime \prime}$ $\mathrm{N}$ and Longitude $1^{\circ} 73^{\prime} 05^{\prime \prime} \mathrm{W}$, with an elevation of $400 \mathrm{~m}$ above sea level. The vegetation is characterized by shrubs and trees surrounding the community but no vegetation in-between houses (Fig. 2). Road puddles that create breeding habitat after rains are common. Stagnant pools from trapped bathroom water at the backyard of houses were also very common and are responsible for breeding nuisance culicines. The houses are built with concrete blocks, with a few mud houses. Doors and windows are hardly screened against mosquitoes. The community has a health post equipped with a microbiology laboratory that has the capacity to diagnose malaria by microscopy. Additionally, there are at least 3 private chemical (pharmaceutical) shops where anti-malarial drugs could be purchased. Inhabitants are mainly farmers, growing subsistence crops such as cassava, maize and plantain proximal to the community which are known to increase the risk of malaria infection by providing resting places for Anopheles mosquitoes [16].

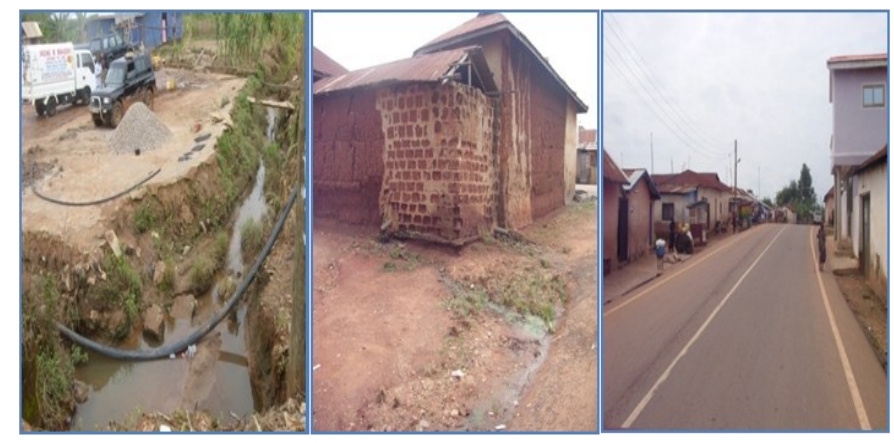

Fig. 2- Photos of parts of Akropong Township 


\section{Atonsu}

Atonsu is a suburb of Kumasi, located in the vicinity of Kumasi South Hospital, N 664' 92" W $1^{\circ} 58^{\prime} 17^{\prime \prime}$. The houses are made up of brick walls and roofed with corrugated iron sheets as well as thatch. Only a few houses have screened doors and windows. Many houses are clustered together with little spaces in-between them. There are very few well planned roads and this gives an impression of poorly planned closed community. Poor drainage system with choked gutters is a common feature. One end of the community is bounded by thick vegetation made of shrubs and trees conducive for mosquito outdoor resting and oviposition with a stream bisecting it. The economic activity in this study area is farming and trading; vegetable farms that use small scale irrigation schemes are also seen. Mosquitoes usually aggregate around houses proximate to these vegetable farms (Fig. 3). Atonsu has previously been described as urban-poor population based on the characteristics of the community [17].

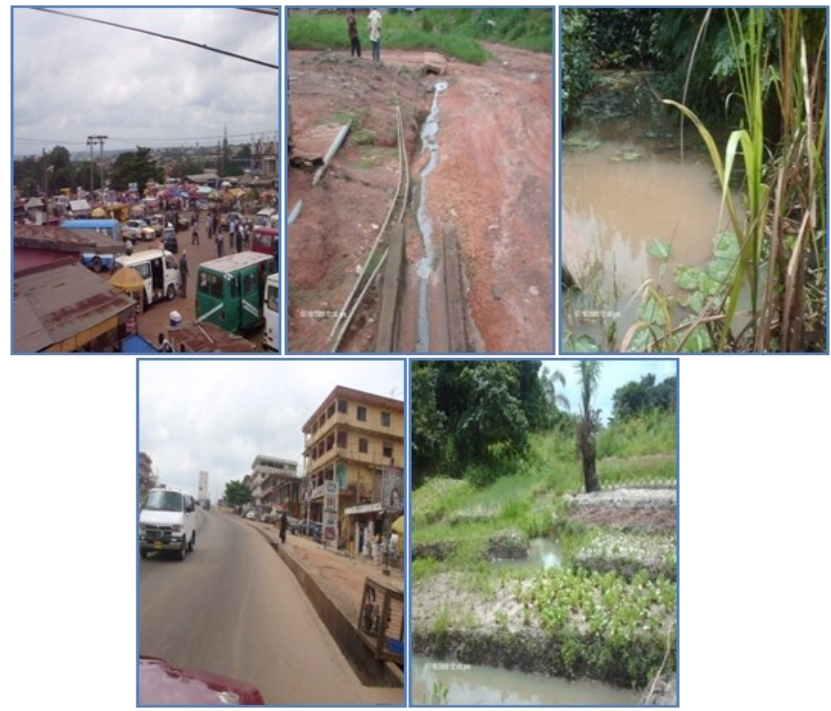

Fig. 3- Photos of parts of Atonsu Township

\section{Emena}

Emena is located off the Kumasi-Accra highway, approximately 15 $\mathrm{km}$ away from the center of Kumasi. It is thus a suburban area of Kumasi; this community exhibits typical characteristics of a periurban area which is rapidly-developing with buildings literally springing up everywhere. However, it is not uncommon to see houses yet under construction but already being occupied by caretaker families (Fig. 4). The majority of the houses are built with concrete blocks with corrugated iron roofing sheets. At Emena, the average number of people sleeping in a room was 3. Majority of the rooms would be susceptible to mosquito entry since there had not been any netting in the windows. Some inhabitants were living in uncompleted buildings and thus had no real doors and windows only cloth were used for this purpose. The surrounding is further characterized by vegetation made up of shrubs, trees, plants and gardens, separating houses. The community has a private hospital (Aninwaa Medical Centre); it is a prominent medical facility in the community. The eastern side of the hospital has a water logged area with sugarcane farms and this is a potential breeding site of mosquitoes.

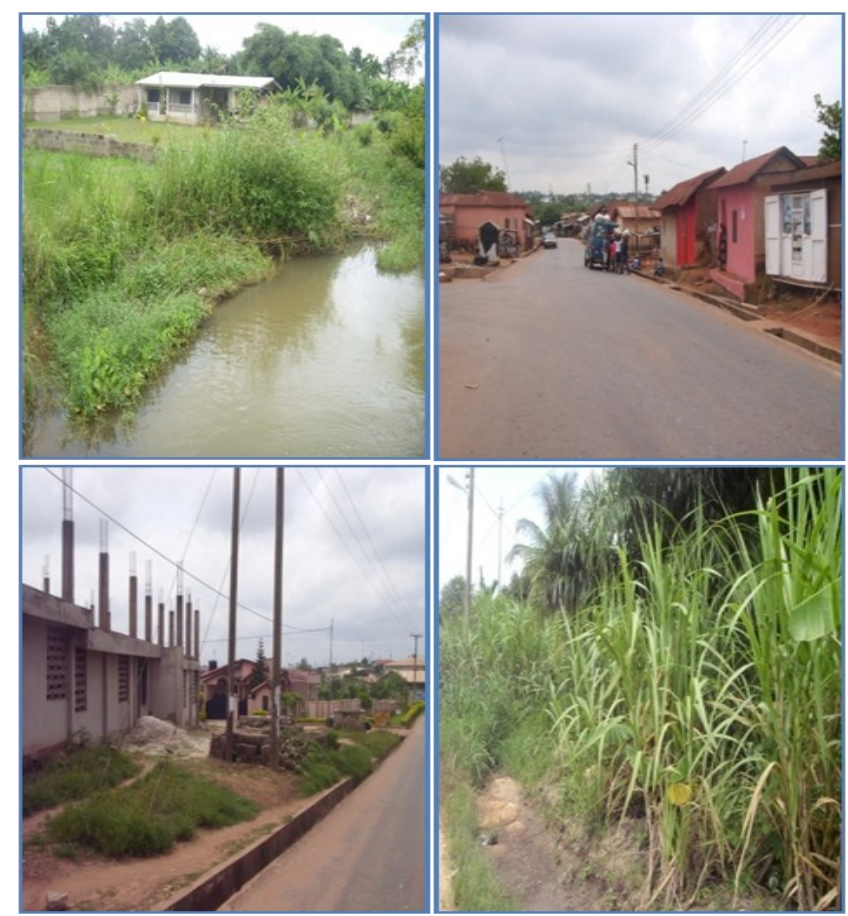

Fig. 4- Photos of Parts of Emena Township

\section{Malaria Data}

Monthly passive case data based on confirmed malaria cases were obtained from the hospitals for the period January-December 2008. However, for the period January-October 2009, this data was obtained on daily basis. These were arranged according to age and gender.

\section{Meteorological Data}

Meteorological data sets, i.e. rainfall, relative humidity and temperature (from Jan 2008-Oct 2009) were obtained from the nearest Ghana Meteorological Services Station for each study area. Data from the weather stations used were from KNUST station covering Aninwa Medical Centre\& Kumasi South Hospital and Owabi station for Akropong Health Centre respectively.

\section{Laboratory Procedures}

Standard procedures in determining malaria cases were applied in all the hospitals in our study sites. Standard procedure by microscopy as described by [18] is the routine procedure in the hospitals. Briefly, samples were collected by the standard finger-prick method and thick and thin smears were prepared. The thin and thick blood smears were air dried. Thin smears were fixed in methanol and thick smears were stained in $4 \%$ Giemsa for 30 minutes. Slides were examined under $\times 100$ oil immersion to identify and count the parasite species. Parasite density was scored against 200 leukocytes when the slide was positive; otherwise, the whole slide was carefully scanned before being declared negative.

\section{Result}

\section{Correlation Between Malaria Cases and Climatic Variability at Akropong}

Malaria cases were reported throughout the year, with a mean number of cases of 107.55 per month (95\% Cl $96.29-118$ ) for the 
period January 2008 to October 2009. The highest malaria incidence of 151 cases was recorded in October, 2008 (Fig. 5). Malaria case incidence correlated significantly with rainfall when the data was staggered to allow for one month lag effect $\left(r^{2}=0.192, p=\right.$
0.041) (Table 1). February 2008 recorded the lowest number of malaria cases of 45 , with total rainfall of $33.6 \mathrm{~mm}$, relative humidity of $49.1 \%$ and a maximum temperature of $34.8^{\circ} \mathrm{C}$ (Fig. 5). This month typically falls within the dry season.

Table 1- Showing the Relationship among Rainfall, Temperature and Relative Humidity

\begin{tabular}{|c|c|c|c|c|c|c|c|c|c|c|c|c|c|}
\hline \multirow{2}{*}{ Rainfall } & \multirow[b]{2}{*}{ Month lag } & \multicolumn{4}{|c|}{ Akropong } & \multicolumn{4}{|c|}{ Atonsu } & \multicolumn{4}{|c|}{ Emena } \\
\hline & & 0 & 1 & 2 & 3 & 0 & 1 & 2 & 3 & 0 & 1 & 2 & 3 \\
\hline & Pearson $r$ & -0.193 & 0.439 & 0.275 & 0.062 & 0.115 & -0.164 & -0.007 & 0.134 & -0.003 & 0.46 & 0.545 & 0.211 \\
\hline & $\mathrm{R}^{2}$ & 0.037 & 0.192 & 0.075 & 0.004 & 0.013 & 0.027 & 0 & 0.018 & 0 & 0.211 & 0.297 & 0.045 \\
\hline Maximum & Pearson $r$ & -0.363 & -0.34 & -0.128 & 0.123 & 0.067 & -0.046 & 0.099 & 0.419 & 0.11 & -0.208 & 0.002 & 0.258 \\
\hline Temp & $\mathrm{R}^{2}$ & 0.132 & 0.115 & 0.016 & 0.015 & 0.004 & 0.002 & 0.01 & 0.175 & 0.012 & 0.043 & 0 & 0.067 \\
\hline & $P$ value & 0.097 & 0.122 & 0.572 & 0.586 & 0.779 & 0.846 & 0.679 & 0.066 & 0.664 & 0.407 & 0.993 & 0.301 \\
\hline Temp & $\mathrm{R}^{2}$ & 0.004 & 0.262 & 0.03 & 0.004 & 0.012 & 0.005 & 0.228 & 0.001 & 0.312 & 0.053 & 0 & 0.002 \\
\hline & $P$ value & 0.769 & 0.015 & 0.443 & 0.772 & 0.65 & 0.766 & 0.033 & 0.922 & 0.016 & 0.356 & 0.953 & 0.874 \\
\hline Relative & Pearson $r$ & 0.36 & 0.485 & 0.276 & 0.107 & -0.18 & 0.024 & 0.206 & -0.328 & -0.35 & -0.137 & -0.029 & -0.151 \\
\hline Humidity & $\mathrm{R}^{2}$ & 0.129 & 0.235 & 0.076 & 0.011 & 0.032 & 0.001 & 0.043 & 0.108 & 0.123 & 0.019 & 0.001 & 0.023 \\
\hline & $P$ value & 0.1 & 0.022 & 0.214 & 0.637 & 0.448 & 0.92 & 0.383 & 0.158 & 0.154 & 0.587 & 0.909 & 0.549 \\
\hline
\end{tabular}

Furthermore, inter annual variation in malaria cases was observed, for e.g. August 2008 recorded 87 cases of malaria as compared to 137 cases in August 2009, although the latter had only a minimal amount of rainfall of $17.9 \mathrm{~mm}$. This may be associated with the heavy rainfall in the preceding months. Linear regression analysis showed a positive correlation with rainfall although this was not significant at the 2-3 month lags $(r=0.275, p 0.216 ; r=0.062, p$ 0.75 respectively) (Table 1 ). A Regression analysis revealed maximum temperature was negatively correlated with malaria cases between time lags of 0-2 months, but increased with increasing malaria cases in the third lag month though not significant (Table 1). The opposite was true for minimum temperature, where it had positive association with malaria cases from 0-2 lag months but not the third. Significant correlation was observed at one month lag time ( $\left.r^{2}=0.2, p=0.015\right)$ (Table 1). Malaria cases generally increased with increasing relative humidity (Fig. 5) and this was statistically significant at the one month lag interval $\left(r^{2}=0.235, p=\right.$ 0.022).

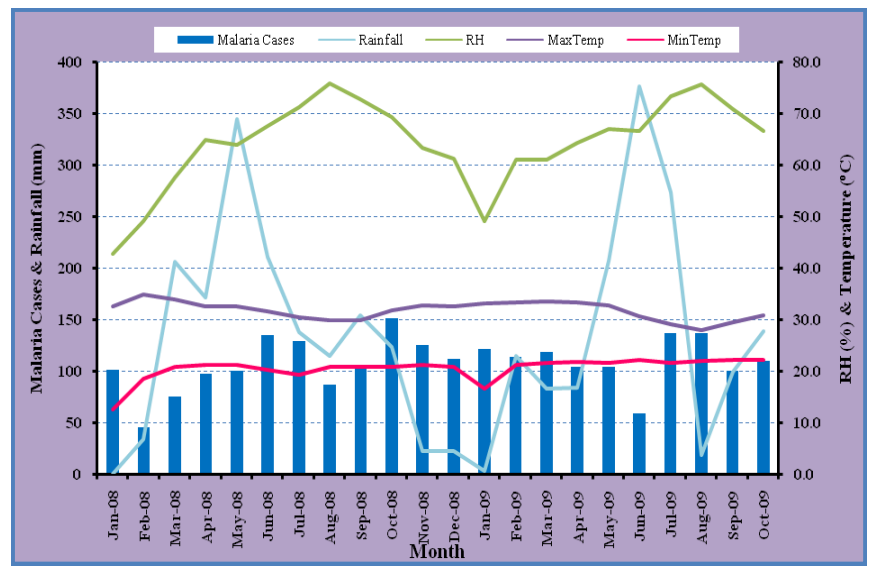

Fig. 5- Climatic variables on malaria incidence (January, 2008 to October, 2009) at Akropong Health Centre

Relationship Between Malaria Cases and Climatic Changes at Atonsu

Malaria cases were reported throughout the study period with an average monthly malaria cases of 104.1 (95\% Cl 89.23-119.0), from January 2008 to August 2009. This is among the highest number of cases observed second only to Akropong, the rural area by only three cases. Regression analysis revealed increasing rainfall results with increasing malaria incidence at 0 and 3 month lag effect, but the opposite was true at 1 and 2 month lag effect although all the correlation was not significant for all the months (Table1). Inter annual variations in rainfall and malaria incidence was observed for e.g., the highest rainfall in both years was recorded in the month of June (Fig. 6). In 2008, this was followed in July by the highest malaria incidence of 166 cases, in 2009 however, even higher rainfall was rather followed by lower malaria incidence. March 2008 recorded the least number of malaria cases of 39 with total rainfall of $97.4 \mathrm{~mm}$ (Fig. 6). This month normally marks the beginning of the wet season and rightly so because the total rainfall was higher than that observed in January and February that same year and is above the $88 \mathrm{~mm}$ needed to favor mosquito breeding.

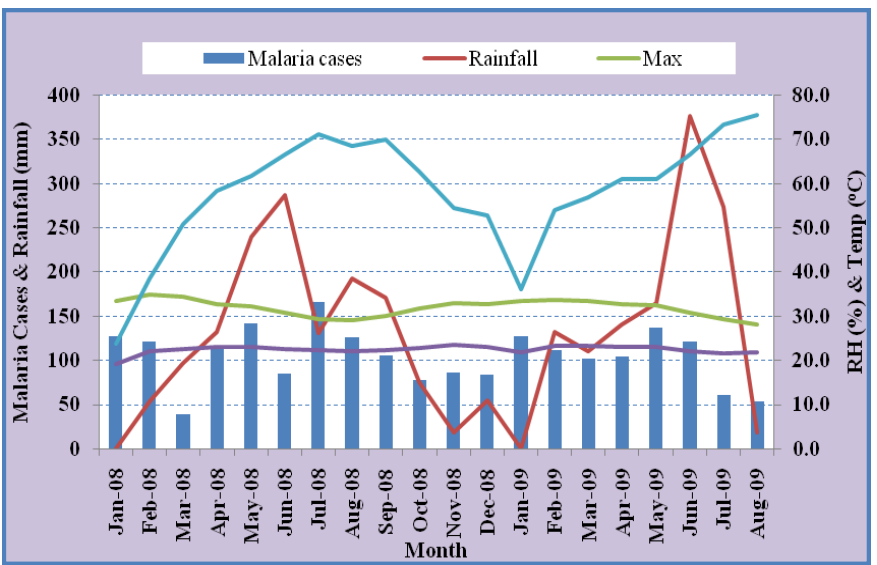

Fig. 6- Climatic variables on malaria incidence (January 2008 to August 2009 at Kumasi South Hospital, Atonsu)

However, March 2009 recorded 101 malaria cases with a total rainfall of $110.6 \mathrm{~mm}$. August 2008 recorded 126 cases of malaria as compared to 53 cases in August 2009, which experienced a minimal amount of rainfall of $17.9 \mathrm{~mm}$. The low rainfall of $17.9 \mathrm{~mm}$ was not conducive for development of mosquitoes. Temperature ranges were within the acceptable values for mosquito survival and 
parasite development. Although maximum temperature increased with increasing malaria incidence except for the one month lag, regression analysis could not confirm any significant correlation at any time lag. However, minimum temperature was strongly associated with increasing malaria incidence and this was significant at one month lag effect $\left(r^{2} 0.228, p=0.033\right)$ (Table1).

\section{Relationship Between Malaria Cases and Climatic Changes at Emena}

Malaria cases were reported every month of the year, with a mean monthly malaria cases of 43.89 (95\% Cl 31.88-55.90), from January 2008 to June 2009. The highest malaria incidence of 99 cases was recorded in January, 2008 (Fig. 7) which falls within the dry season and thus recorded no rainfall. Over all, the lowest mean monthly malaria case incidence was observed as this site. When the data was staggered to allow for 0-3 month lag effect, logistic regression analysis, revealed that rainfall was negatively correlated with malaria incidence at the zero month lag but positively associated with malaria case incidence at one to three month lags and this was significant at the two month lag $\left(r^{2}=0.297 p=0.018\right)$. Maximum temperature generally had weak positive correlation with malaria except at one month lag where it had an inverse correlation, but all these correlation was not statistically significant (Table 1).

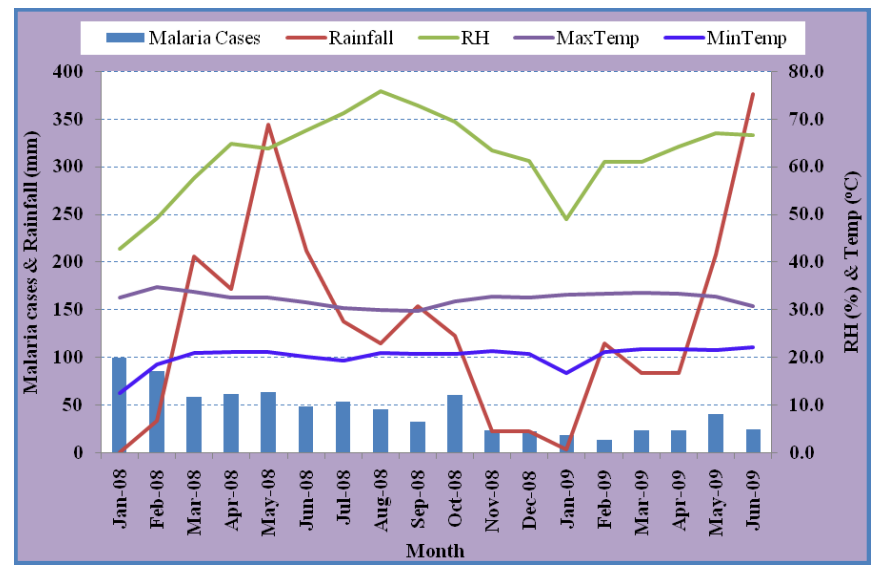

Fig. 7- The effects of climatic variables on malaria incidence (blue bars) observed for the period January 2008 to June 2009 at Emena Hospital located in a peri-urban area of Kumasi.

Minimum temperature was negatively associated with malaria cases at this site and this was significant at zero month lag $(r-0.559$, $p=0.016)$. Increasing relative humidity correlated negatively with malaria cases at all time lags but this correlation was weak as there was not statistical significance at any time lag (Table 1). Inter annual variation in case numbers was also observed at this site, for e.g. February 2009, the least number of malaria cases of 13 was observed with total rainfall of $114.3 \mathrm{~mm}$, relative humidity of $61.0 \%$ and a maximum temperature of $33.3^{\circ} \mathrm{C}$ (Fig. 7). This month normally falls within the dry season but this time around, the rainfall recorded was markedly high. This could flood mosquito breeding sites and wash away mosquito larvae and kill them. The relative humidity was above the optimal value of $\geq 60 \%$, with the maximum temperature outside the optimal value of $30^{\circ} \mathrm{C}$.

Furthermore, February 2008 recorded 85 cases of malaria as compared to 13 cases in February 2009 with February 2008 having a total rainfall of $33.6 \mathrm{~mm}$ compared to 114.3 in February 2009.

\section{Comparison of Mean Monthly Malaria Cases, Rainfall, Relative Humidity and Monthly Temperature among the Study Sites.}

\section{Malaria cases}

Mean monthly malaria cases at the three study sites differed significantly $(F=32.49, p<0.0001)$ with the highest from the rural area Akropong, followed by Atonsu, the urban area and then Emena, the peri-urban area. However post hoc analysis (HSD Tukey multiple comparison) revealed no statistical difference between Atonsu and Akropong ( $q=0.57, p>0.05)$.

\section{Rainfall}

Mean monthly rainfall was not significally different from all the study sites compared with each other $(F=0.0014, p=0.9986)$. However, total annual rainfall was highest at Akropong (2936.4mm), followed by Atonsu $(2666.2 \mathrm{~mm})$ and then Emena $(2407.1 \mathrm{~mm})$ recorded the least. Interestingly, total number of absolute malaria cases followed a similar trend.

\section{Relative Humidity}

Comparison of relative humidity across the study sites revealed no significant differences statistically $(F=1.874, p=0.1628)$. This is in spite of Atonsu (urban) showing a lower overall mean RH: Atonsu $58.22 \%(95 \% \mathrm{Cl} 52-64)$, Akropong 64.28\% (60-68) and Emena $62.66 \%(58-66)$.

\section{Mean Temperature}

Mean monthly temperature observed at the three study sites were significantly different from each other $(F=4.041, p=0.023)$. With Atonsu on the average was $1^{\circ} \mathrm{C}$ warmer than the other sites: Atonsu $27.08^{\circ} \mathrm{C}(95 \% \mathrm{Cl}, 26.55-27.61)$, Akropong $26.10^{\circ} \mathrm{C}(95 \%$ $\mathrm{Cl} 25.58-26.63)$ and Emena $26.24^{\circ} \mathrm{C}(95 \% \mathrm{Cl} 25.62-26.86)$.

\section{Discussion}

It has long been established that climate is an important determinant of vector and pathogen distribution [19]. Because the biology of malaria transmission makes it very sensitive to changes in climate [20] and thus Climate variability has a strong impact on malaria transmission in space and time [20]. Less known is the specific role of climatic variability on malaria incidence across differing micro-epidemiological settings at the local scale and its utility in early warning systems for the management of local malaria burden [21].

Mean monthly rainfall was comparable at all study sites with no differences statistically. However, the total amount of annual rainfall was highest at Akropong, followed by Atonsu and then by Emena. It is interesting to note that the number of malaria cases followed a similar trend. Generally, increasing rainfall was associated with increasing malaria cases; however this differed with particular micro-epidemiological setting. At the rural microepidemiological setting, rainfall had a strong positive correlation with malaria at one month lag, but at the peri-urban area, this strong positive correlation occurred at two month lag after the rains. At the urban site however, malaria cases had only weak correlation with rainfall at different month lags, there was a positive correlation at zero and three-month lag but negative correlation at 
one and two month lags but these correlations were not statistically significant. From the north-eastern part of the forest zone of Ghana, a recent investigation on temporal associations between weekly malaria incidence and weekly rainfall, found that the level of rainfall predicted the malaria incidence after a mean time lag of two months [13], our findings are comparable to this study. Similarly, several other studies found direct correlation between rainfall and malaria incidence [22-24].

In contrast, others found negative correlation between rainfall and malaria incidence [25-26], whilst others observed weak correlations between rainy days and the incidence of malaria [27]. A study found that there was a high spatial variation in the sensitivity of malaria outpatient number to climate fluctuations in the highlands of Eastern Africa [14]. However, another study observed that seasonally varying effects of rainfall on malaria case counts may explain weak overall cross-correlations [28].

Our observation in keeping with other observations yield to the phenomenon that the impact of climatic variables on the incidence of malaria is not homogenous. Thus, it is important that spatial and seasonal variation in micro epidemiological settings should be taken into account in malaria predictive models using rainfall as covariates.

Maximum temperature had a weak correlation with malaria case incidence at all three sites studied. At the rural setting, it was inversely correlated with malaria from 0-2 month lag but a positive correlation at the third month lag, this though was not significant. However, at the peri-urban and urban sites, except at one month lag where it has a negative correlation, maximum temperature positively correlated with malaria and this relationship was weak and not significant. Mean monthly temperature observed at the three sites were different from each other significantly. Atonsu on the average was $1^{\circ} \mathrm{C}$ higher than the others. Minimum temperature appeared to be very important factor distinguishing between the various sites in terms of its influence on malaria incidence. It was significantly associated with malaria incidence at one month lag at the rural site, at two month lag at the peri-urban area. However, it was negatively associated with malaria case incidence at the urban area that has $1^{\circ} \mathrm{C}$ elevated temperature. The results of similar study conducted in China [23] and in India [24] suggested a strong positive correlation between monthly incidence of malaria and monthly maximum and minimum temperature.

Temperature plays an important role in the variability of malaria transmission by regulating the development rate of mosquito larvae and influencing the survival rate of adult mosquitoes (Martens et al., 1995). Higher temperatures $\left(30^{\circ} \mathrm{C}\right)$ increases the number of times eggs are laid by mosquitoes [6] and they generally develop faster and feed (blood meals) earlier in their life cycle [27]. At $16^{\circ} \mathrm{C}$, larval development may take more than 45 days (reducing the number of mosquito generations and putting the larvae at increased risk of predators) [8]. Below $20^{\circ} \mathrm{C}$, the life cycle of $P$. falciparum is limited [29].

Relative humidity was positively correlated with malaria at the rural setting and significantly so at one month lags. On the other hand, it was negatively correlated with malaria at the urban setting. However, it had weak positive and negative correlations with malaria at the peri-urban area at different time lags. Correlation between hu- midity and malaria incidence has a direct relationship whiles that between maximum temperature and malaria incidence gave an indirect relationship. Temperatures of $20^{\circ} \mathrm{C}$ to $30^{\circ} \mathrm{C}$ and humidity greater than $60 \%$ are optimal for Anopheles to survive long enough to acquire and transmit the parasite [23].

Besides climatic factors, there are many variables that affect malaria transmission, other non-climatic factors such as nearby watercourses, vegetation index and soil type in the immediate surroundings [28]. Other factors include vector species, human activities, socio-economic conditions like developmental changes such as construction, housing and living conditions, adopted control measures, local ecological environment, suitability for mosquito vector (vegetation, introduction of irrigation schemes) and drug resistance in malaria parasites [24]. Akropong being a rural community has majority of its residents being farmers and they normally farm close to their homes.

Atonsu has a characteristic of an urban poor community [17]. The complex factors contributing to malaria risk in urban areas are not fully understood but evidence is rapidly accumulating that the urban poor are at far higher risk from malaria than previously acknowledged [30]. This is in accordance with findings at Atonsu, an urban setting that has similar malaria burden like the rural community in our study. This may be due to several factors including open eaves without ceiling allowing easy mosquito entry and inability to afford indoor residual insecticides. Again, farming as well as commercial activities contribute to the creation of insect-breeding sites [30]. Although the maximum temperature was slightly above the optimal value of $30^{\circ} \mathrm{C}$, both the total rainfall and the relative humidity favored mosquito-breeding which contributed to the high number of malaria cases.

\section{Conclusion}

We have investigated the relationship between climatic variables and malaria case incidence in three eco-epidemiological settings; rural, peri-urban and urban areas in the forest zone of Ghana and have observed different effects of rainfall, temperature and relative humidity on the incidence of malaria in these settings.

The result of this study indicates that climatic variables scale can be useful and are relevant for malaria forecasting and control. Knowledge of climate variability and its effect on the incidence of malaria on a micro geographic scale has the potential to be useful to predict malaria outbreak before its occurrence, either to strengthen control measures enough to prevent the outbreaks or at least to create adequate facilities for the appropriate treatments.

\section{Recommendation}

Further study is needed over a longer period to validate the results of the present study. As long-term weather forecasting becomes more accurate, it may even be possible to forecast the weather that might trigger an outbreak. It is also recommended that there should be more collaboration between malaria epidemiologist and climatologist to foster the use of mutual information on malaria indices and climatic data to foster the development of strong systems of surveillance and early warning systems to prevent epidemics.

\section{Author's Contribution}

DCM conducted the field survey and collected laboratory data, 
SCKT, AM and SKD developed the study protocol and supported field operations. ; KB, GA performed data analysis and drafted the manuscript; HHA, AT and HAH participated in the study design and implementation; SCKT, SKD, CC and AM conceived the study. All authors read and approved the final manuscript.

\section{Acknowledgement}

This project was carried out as part of the African Monsoon Multidisciplinary Analysis (AMMA) project of the European Union (EU). We therefore acknowledge the financial support provided by the AMMA project for the execution of the research work. We also thank the College of Science, KNUST, for the assistance it provided in hosting and administering the AMMA project.

\section{References}

[1] Martens P., Hall L. (2000) Emerg. Infect. Dis., 6, 28-45.

[2] Rowe A.K., Rowe S.Y., Snow R.W. (2006) Inter. J. Epid., 35, 691-704.

[3] World Health Organization (2009) World Malaria Report, Geneva, Switzerland.

[4] Abonuusum A., Owusu-Daako K., Tannich E., May J., Garms R., Kruppa T. (2011) Parasitol Res., 108, 1465-147

[5] De graft A.A., Agyei-Mensah S. (2007) Epidemiological transition and the double burden of disease in Accra, Ghana.

[6] Martens W.J. (1995) Med. War., 11, 202-13.

[7] Patz J., AMcGeehin M.A., Bernard S.M., Ebi K.L., Epstein P.R., Grambsch A., Gubler D.J., Reither P., Romieu I., Rose J.B., et al. (2000) Environ. Health Perspective, 108, 367-376.

[8] Teklehaimanot H.D., Lipsitch M., Teklehaimanot A., Schwartz J. (2004) Malar J., 3, 41.

[9] Craig M.H., Kleinschmidt I., Nawn J.B., Le Sueur D., Sharp B.L. (2004) Trop. Med. Int. Health, 9, 1247-1257.

[10]Worrall E., Connor S.J., Thomson M.C. (2007) Trop. Med. Int. Health, 12, 75-88.

[11]Van der Hoek W., Konradsen F., Perera D., Amerasinghe P.H., Amerasinghe F.P. (1997) Ann. Trop. Med. Parasitol., 91, 945949.

[12]De Alwis R., Wijesundere A., Ramasamy M.S., Ramasamy R. (1990) Current Status of Malaria Research in Sri Lanka Eds. Ramasamy R. Kandy, Institute of Fundamental Studies, 80-84.

[13]Krefis A.C., Schwarz N.G., Nkrumah B., Acquah S., Loag W. (2011) PLOS ONE 6(3), e17905.

[14]Zhou G., Minakawa N., Githeko A.K., Yan G. (2004) Proc. Nat. Acad. Sci., USA, 101(8), 2375-80.

[15]Ghana Statistical Service (2003) Centre for Health Information Management.

[16]Krefis A.C., Schwarz N.G., Krüger A., Fobil J., Nkrumah B., Acquah S., Loag W., Sarpong N., Adu-Sarkodie Y., Ranft U., May J. (2011) Am. J. Trop. Med. Hyg., 84, 285-291.
[17]Tay S.C.K., Danuor S.K., Morse A., Caminade C., Badu K. (2011) Journal of Environmental Science and Engineering, USA (in Press).

[18]Slutsker L., Taylor T., Wirima J., Steketee R. (1994) Trans. R. Soc. Trop. Med. Hyg., 88, 548-51.

[19]Gill C.A. (1921) Indian J. Med. Res., 8, 633-93.

[20]Githeko A.K. (2009) Commonwealth Health Ministers' Update, 42-45.

[21]Yé Y., Louis V.R., Simboro S. and Sauerborn R. (2007) BMC Public Health, 7, 101.

[22]Kilian A.H.D., Langi P., Talisuna A., Kabagambe G. (1999) Trans R. Soc. Trop. Med. Hygiene, 93, 22-23.

[23]Bi P., Tong S., Donald K., Parton K.A., Ni J. (2003) Public Health Rep. 118, 65-71.

[24]Pemola N.D. and Jauhari R.K. (2006) India. J. Vect. Borne Dis., 43, 21-28.

[25]Paulo L.O, Antonio P.R, Alina R.V, Nicolás L.V. and Manuel D.G. (1997) Environmental Health Prospects.

[26]Singh N. and Sharma V.P. (2002) Ann. Trop. Med. Parasitol., 96(4), 349-59.

[27]Grover-Kopec E.K., Blumenthal M.B., Ceccato P., Dinku T., Omumbo J.A. and Connor S.J. (2006) Africa. Malaria Journal, $5(38)$.

[28]Cottrell G., Kouwaye B., Pierrat C., le Port A., Bouraı"ma A., et al. (2012) PLoS ONE 7(1), e28812.

[29]McMichael A.J. and Martens W.J.M. (1995) Eco. Syst. Health, 1, 23-33.

[30]Klinkenberg E., McCall P.J., Hastings I.M., Wilson M.D., Amerasinghe F.P. and Donnelly M.J. (2005) Emerg. Infect. Dis., 11, 1290-1293. 